\title{
The Number of Partitions of the Integer $N$ into $M$ Nonzero Positive Integers
}

\author{
By W. J. A. Colman
}

Abstract. The function $p_{m}(n)$ is defined as the number of partitions of the integer $n$ into exactly $m$ nonzero positive integers where the order is irrelevant.

A series in which the leading terms alternate in sign is given for $p_{m}(n)$ which yields good numerical estimates.

1. Introduction. If $p_{m}(n)$ is the number of partitions of the integer $n$ into exactly $m$ positive integers and if $p_{m}^{*}(n)$ is the number of partitions into at most $m$ parts and $p(m)$ is the usual partition function, then there are some simple known relationships between them.

$$
\begin{aligned}
p_{m}(n)-p_{m}(n-m) & =p_{m-1}(n-1), \\
p_{m}^{*}(n) & =p_{m}(n+m), \\
p(m) & =p_{m}(2 m) .
\end{aligned}
$$

Closed formulae for $p_{m}(n)$ are known for small $m$; see Gupta. In particular, we have

$$
\begin{gathered}
p_{2}(n)=\left[\frac{n}{2 ! 1 !}\right], \quad p_{3}(n)=\left[\frac{n^{2}+3}{3 ! 2 !}\right], \\
p_{4}(n)=\left[\frac{n^{3}+3 n^{2}+\frac{1}{2}\left\{9 n(-1)^{n}-9 n\right\}+32}{4 ! 3 !}\right] .
\end{gathered}
$$

The formulae for $m=2$ and $m=3$ are well known and the formula for $m=4$ is equivalent to a formula given by A. De Morgan (Dickson [2, p. 115]). Thus $p_{m}(n)$ is not a polynomial, but it contains a completely algebraic part which is a polynomial in $n$ of degree $(m-1)$. If we call these polynomials $q_{m}(n)$ (say), then they satisfy the relationship

$$
q_{m}(n)-q_{m}(n-m)=q_{m-1}(n-1)
$$

2. A Series Expansion for $q_{m}(n)$. By writing $q_{m}(n)=a_{m 1} n^{m-1}+a_{m 2} n^{m-2}+$ $a_{m 3} n^{m-3}+\ldots$ and substituting in the recurrence relationship above, we can equate powers of $n$ to yield the relationship

$$
\begin{aligned}
b_{m r}= & \frac{(r-1)}{r !} \sum_{k=1}^{m} b_{k 1} k^{r-1}+\frac{(r-2)}{(r-1) !} \sum_{k=1}^{m} b_{k 2} b^{r-2}+\cdots+\frac{1}{2 !} \sum_{k=1}^{m} b_{k r-1} k \\
& -\left(\frac{b_{m 1} m^{r-1}}{(r-1) !}+\cdots+\frac{b_{m r-1} m}{1 !}\right),
\end{aligned}
$$

where $a_{m r}=m !(m-r) ! b_{m r}$ so that, as $a_{m 1}=1 / m !(m-1) !$, we have $b_{m 1}=1$.

Received January 25, 1980; revised April 13, 1981 and November 17, 1981

1980 Mathematics Subject Classification. Primary 05A17, 39A30. 
This formula can be used to successively determine the $a_{m r}$ to give the leading coefficients of $q_{m}(n)$. We have

$$
a_{m 2}=\frac{m(m-3)}{4 m !(m-2) !}, \quad a_{m 3}=\frac{m^{4}-\frac{58 m^{3}}{9}+\frac{75 m^{2}}{9}-\frac{2 m}{9}}{4^{2} \cdot 2 ! m !(m-3) !} .
$$

This leads to the expansion

$$
\begin{aligned}
q_{m}(n)= & \frac{n^{m-1}}{m !(m-1) !}+\frac{1}{m !(m-2) !}\left(\frac{m-3 m}{4 \cdot 1 !}\right) n^{m-2} \\
& +\frac{1}{m !(m-3) !}\left(\frac{m^{4}-\frac{58 m^{3}}{9}+\frac{75 m^{2}}{9}-\frac{2 m}{9}}{4^{2} \cdot 2 !}\right) n^{m-3} \\
& +\frac{1}{m !(m-4) !}\left(\frac{m^{6}-\frac{31 m^{5}}{3}+29 m^{4}-\frac{65 m^{3}}{3}+2 m^{2}}{4^{3} \cdot 3 !}\right) n^{m-4}+\frac{1}{m !(m-5) !} \\
& \cdot\left(\frac{m^{8}-14 \frac{2}{3} m^{7}+66 \frac{16}{27} m^{6}-107 \frac{29}{225} m^{5}+55 \frac{134}{135} m^{4}-10 \frac{54}{135} m^{3}+\frac{4}{27} m^{2}-\frac{16}{225} m}{4^{4} \cdot 4 !}\right) n^{m-5} .
\end{aligned}
$$

The polynomials $b_{m r}$ can be generated by means of a computer program where the summations are effected using the Bernoulli polynomials. This expansion although of some interest is of little use for calculating $p_{m}(n)$ unless $n$ is very large compared with $m$.

G. J. Rieger has given the over estimate

$$
p_{m}(n)<\frac{1}{m !(m-1) !}\left(n+\frac{m(m-3)}{4}\right)^{m-1} \text { for } m \geqslant 4,
$$

which exactly agrees with the first two terms of the expansion. This yields good estimates when $n$ is large compared with $m(m-3) / 4$ but gives no idea of the magnitude of the error. To determine a series expansion which will yield better numerical approximations and give some idea of the magnitude of the error, we can proceed as follows.

3. An Alternating Series Expansion. Assume there is an expansion of the form

$$
q_{m}(n)=\sum_{r=1,3,5} \frac{F_{r}(m)}{m !(m-r) !}\left(n+\frac{m(m-3)}{4}\right)^{m-r} \quad \text { where } F_{1}(m)=1,
$$

we have

$$
q_{m}(n-m)=\sum \frac{F_{r}(m)}{m !(m-r) !}\left(n+\frac{m(m-3)}{4}-m\right)^{m-r}
$$

and

$$
q_{m-1}(n-1)=\sum \frac{F_{r}(m-1)}{(m-1) !(m-r-1) !}\left(n+\frac{m(m-3)}{4}-\frac{m}{2}\right)^{m-r-1} .
$$

Write

$$
q_{m}(n)=\sum \frac{F_{r}(m)}{m !(m-r) !}\left(n+\frac{m(m-3)}{4}-\frac{m}{2}+\frac{m}{2}\right)^{m-r}
$$


and

$$
q_{m}(n-m)=\sum \frac{F_{r}(m)}{m !(m-r) !}\left(n+\frac{m(m-3)}{4}-\frac{m}{2}-\frac{m}{2}\right)^{m-r} .
$$

Put $X=n+m(m-3) / 4-m / 2$ (say) and expanding both sides in powers of $X$ and equating, we have

$$
\frac{F_{1}(m) m^{r-1}}{r ! 2^{r-1}}+\frac{F_{3}(m) m^{r-3}}{(r-2) ! 2^{r-3}}+\cdots+\frac{F_{r}(m) m^{0}}{1 ! 2^{0}}=F_{r}(m-1)
$$

where $F_{1}(m)=1$ and $r=3,5,7, \ldots$,

$$
\therefore F_{r}(m)-F_{r}(m-1)=-\left(\frac{F_{1}(m) m^{r-1}}{r ! 2^{r-1}}+\frac{F_{3}(m) m^{r-3}}{(r-2) ! 2^{r-3}}+\cdots+\frac{F_{r-2}(m) m^{2}}{3 ! 2^{2}}\right) \text {. }
$$

Hence the $F_{r}(m)$ can be determined sequentially, and the above expansion is possible. Now $F_{r}(m)$ is a polynomial in $m$ of degree $(3 r-3) / 2$ without constant term.

Thus $F_{r}(0)=0$. Putting $m=0$, we have immediately that $F_{r}(-1)=0$. Putting $m=-1$ yields $F_{r}(-2)=1 / r ! 2^{r-1}$. These values can be used as a check when explicitly calculating the $F_{r}(m)$. We have

$$
\begin{gathered}
F_{r}(m)-F_{r}(m-1)=-\left(\frac{F_{1}(m) m^{r-1}}{r ! 2^{r-1}}+\cdots+\frac{F_{r-2}(m) m^{2}}{3 ! 2^{2}}\right), \\
\vdots \\
F_{r}(1)-F_{r}(0)=-\left(\frac{F_{1}(1) 1^{r-1}}{r ! 2^{r-1}}+\cdots+\frac{F_{r-2}(1) 1^{2}}{3 ! 2^{2}}\right) .
\end{gathered}
$$

Adding we have as $F_{r}(0)=0$ that

$$
-F_{r}(m)=\sum_{i=1}^{m}\left(\frac{F_{1}(i) i^{r-1}}{r ! 2^{r-1}}+\cdots+\frac{F_{r-2}(i) i^{2}}{3 ! 2^{2}}\right), \quad r=3,5,7, \ldots \text { with } F_{1}(m)=1 .
$$

By assuming an expansion of the form

$$
F_{r}(m)=a_{r 1} m^{(3 r-3) / 2}+a_{r 2} m^{(3 r-5) / 2}+a_{r 3} m^{(3 r-7) / 2}+\ldots,
$$

it is easy but tedious to show that

$$
\begin{aligned}
F_{r}(m)= & \frac{(-1)^{(r-1) / 2}}{6^{r-1} 2^{(r-1) / 2}((r-1) / 2) !} \\
& \times\left(m^{(3 r-3) / 2}+\frac{3(r-1)(3 r+16) m^{(3 r-5) / 2}}{100}\right. \\
& \left.\quad+\frac{(r-1)}{49 \times 20,000}\left(3969 r^{3}+42615 r^{2}-39276 r-127870\right) m^{(3 r-7) / 2}+\ldots\right) .
\end{aligned}
$$

Thus the polynomials $F_{r}(m)$ alternate in sign. We have

$$
\begin{aligned}
& F_{1}(m)=1, \\
& F_{3}(m)=-\left(\frac{m^{3}+\frac{3 m^{2}}{2}+\frac{m}{2}}{6^{2} \cdot 2 \cdot 1 !}\right),
\end{aligned}
$$




$$
F_{5}(m)=\left(\frac{m^{6}+\frac{372 m^{5}}{100}+\frac{505 m^{4}}{100}+\frac{270 m^{3}}{100}+\frac{25 m^{2}}{100}-\frac{12 m}{100}}{6^{4} \cdot 2^{2} \cdot 2 !}\right)
$$

These polynomials can again be determined sequentially. The computer printout is given below

$$
\begin{aligned}
& \underline{r}=5 \\
& -0.12 \\
& 0.25 \\
& \begin{array}{l}
2.7 \\
5.05
\end{array} \quad \therefore F_{5}(m)=\frac{1}{6^{4} \cdot 2^{2} \cdot 2 !}\left(m^{6}+3.72 m^{5}+5.05 m^{4}+2.7 m^{3}\right. \\
& 3.72 \\
& 1 \\
& \left.+0.25 m^{2}-0.12 m\right) \\
& \underline{r}=7 \\
& -0.293878 \\
& 0.18 \\
& \begin{array}{l}
2.47214 \\
-2.565
\end{array} \quad \therefore F_{7}(m)=\frac{-1}{6^{6} \cdot 2^{3} \cdot 3 !}\left(m^{9}+6.66 m^{8}+18.6533 m^{7}+26.8264 m^{6}\right. \\
& -18.3964 \\
& -26.8264 \\
& -18.6533 \\
& -6.66 \\
& +18.3964 m^{5}+2.565 m^{4}-2.47214 m^{3} \\
& -1 \\
& \left.-0.18 m^{2}+0.293878 m\right) \\
& \begin{array}{l}
r=9 \\
-2.22171 \\
0.630955
\end{array} \\
& \underline{r=11} \\
& \begin{array}{r}
-36.7225 \\
5.90694
\end{array} \\
& \underline{r=13} \\
& 16.3947 \\
& -4.82027 \\
& -44.5329 \\
& 23.1309 \\
& 157.976 \\
& 258.189 \\
& -36.4973 \\
& -602.259 \\
& 68.4204 \\
& 196.713 \\
& 126.587 \\
& 806.053 \\
& -186.351 \\
& 47.8683 \\
& -1638.34 \\
& -1839.7 \\
& 10.32 \\
& -1104.07 \\
& 1 . \\
& -416.03 \\
& -100.809 \\
& -14.7 \\
& -1 \\
& -1118 \\
& 115.03 \\
& 7682.71 \\
& -637.779 \\
& -16846 \\
& 698.096 \\
& -19211.2 \\
& 137.842 \\
& -15196.3 \\
& 784.916 \\
& 19823.1 \\
& 20845 \\
& 11813.2 \\
& 4357.69 \\
& 1099
\end{aligned}
$$




$\begin{array}{cccc}r=15 & r=17 & \underline{r=19} & \underline{r}=21 \\ -56378.7 & -0.43752 \mathrm{E} 7 & -0.49478 \mathrm{E} 9 & -0.781715 \mathrm{E} 11 \\ 4028.38 & 230048 & 0.199698 \mathrm{E} 8 & 0.25002 \mathrm{E} 10 \\ 382554 & 0.294572 \mathrm{E} 8 & 0.33141 \mathrm{E} 10 & 0.521721 \mathrm{E} 12 \\ -20984.3 & -0.115394 \mathrm{E} 7 & -0.97816 \mathrm{E} 8 & -0.120565 \mathrm{E} 11 \\ -812491 & -0.613873 \mathrm{E} 8 & -0.682167 \mathrm{E} 10 & -0.106477 \mathrm{E} 13 \\ 14732.7 & 548128 & 0.328379 \mathrm{E} 8 & 0.296624 \mathrm{E} 10 \\ 866493 & 0.631721 \mathrm{E} 8 & 0.686827 \mathrm{E} 10 & 0.105652 \mathrm{E} 13 \\ 30820.2 & 0.235611 \mathrm{E} 7 & 0.229987 \mathrm{E} 9 & 0.305372 \mathrm{E} 11 \\ -583067 & -0.397167 \mathrm{E} 8 & -0.416288 \mathrm{E} 10 & -0.625932 \mathrm{E} 12 \\ -55699 & -0.388232 \mathrm{E} 7 & -0.354982 \mathrm{E} 9 & -0.451736 \mathrm{E} 11 \\ 301160 & 0.174792 \mathrm{E} 8 & 0.171547 \mathrm{E} 10 & 0.249008 \mathrm{E} 12 \\ 21659.1 & 0.288588 \mathrm{E} 7 & 0.257655 \mathrm{E} 9 & 0.317883 \mathrm{E} 11 \\ -272765 & -0.626974 \mathrm{E} 7 & -0.525898 \mathrm{E} 9 & -0.718084 \mathrm{E} 11 \\ -276413 & -0.10408 \mathrm{E} 7 & -0.118078 \mathrm{E} 9 & -0.141378 \mathrm{E} 11 \\ -149698 & 0.418598 \mathrm{E} 7 & 0.136369 \mathrm{E} 9 & 0.159685 \mathrm{E} 11 \\ -53845.3 & 0.418626 \mathrm{E} 7 & 0.335923 \mathrm{E} 8 & 0.447758 \mathrm{E} 10 \\ -13719.5 & 0.218906 \mathrm{E} 7 & -0.705382 \mathrm{E} 8 & -0.307064 \mathrm{E} 10 \\ -2504.71 & 768379 & -0.711215 \mathrm{E} 8 & -0.997364 \mathrm{E} 9 \\ -318.096 & 195323 & -0.362286 \mathrm{E} 8 & 0.128808 \mathrm{E} 10 \\ -25.62 & 36850.2 & -0.124395 \mathrm{E} 8 & 0.13368 \mathrm{E} 10 \\ -1 & 5128.87 & -0.313772 \mathrm{E} 7 & 0.668514 \mathrm{E} 9 \\ & 506.447 & -600883 & 0.225198 \mathrm{E} 9 \\ & 32.16 & -87931.4 & 0.562438 \mathrm{E} 8 \\ & 1 & -9680.46 & 0.108307 \mathrm{E} 8 \\ & & -766.529 & 0.163087 \mathrm{E} 7 \\ & & -39.42 & 191397 \\ & & -1 & 17134.1 \\ & & & 1114.23 \\ & & & 47.4 \\ & & & 1\end{array}$

All the polynomials to $r=21$ are of constant sign for $m \geqslant r$. This series has G. J. Rieger's estimate as its first term and being an alternating series will clearly yield successively both over and under estimates for $p_{m}(n)$. Some numerical examples:

Magnitude of each term
(1) $P_{20}(200)=.874388 \times 10^{11}$
$.148195 \times 10^{12}$
$.746174 \times 10^{11}$
$.148195 \times 10^{12}$ Rieger estimate
$.154646 \times 10^{11}$
$.735774 \times 10^{11}$
$.170826 \times 10^{10}$
$.890421 \times 10^{11}$
$.108904 \times 10^{9}$
$.873338 \times 10^{11}$
$.405826 \times 10^{7}$
$.874427 \times 10^{11}$
85496.6
$.874387 \times 10^{11}$
$.874388 \times 10^{11}$ 
The expansion in terms of the $F_{r}(m)$ for $p_{m}(n)$ when truncated at $r=1,3,5$, or 7 gives upper and lower bounds for $p_{m}(n)$ better than those given previously for $n$ tending to infinity.

$$
\begin{array}{rlr}
\text { (2) } P_{20}(500)= & .112794 \times 10^{18} & \\
& .127275 \times 10^{18} & .127275 \times 10^{18} \\
& .152099 \times 10^{17} & .112065 \times 10^{18} \\
& .748178 \times 10^{15} & .112813 \times 10^{18} \\
& .196154 \times 10^{14} & .112793 \times 10^{18} \\
& .296801 \times 10^{12} & .112794 \times 10^{18} \\
& .262506 \times 10^{10} & .112794 \times 10^{18} \\
\text { (3) } P_{30}(1000)= & .716051 \times 10^{26} & \\
& .895919 \times 10^{26} & .895919 \times 10^{26} \\
& .198200 \times 10^{26} & .697718 \times 10^{26} \\
& .194009 \times 10^{25} & .717119 \times 10^{26} \\
& .110784 \times 10^{24} & .716011 \times 10^{26} \\
& .409716 \times 10^{22} & .716052 \times 10^{26} \\
& .103050 \times 10^{21} & .716051 \times 10^{26} \\
& .180151 \times 10^{19} & .716051 \times 10^{26}
\end{array}
$$

4. The Relationship Between $N$ and $M$ for Effective Calculation of $p_{m}(n)$. Thus the series gives excellent results when $n$ is suitably large compared with $m$. In fact this will be achieved if the terms decrease in absolute magnitude. A condition for this can be estimated approximately as follows. We have, apart from sign,

$$
\begin{aligned}
F_{r}(m)= & \frac{1}{6^{r-1} 2^{(r-1) / 2}((r-1) / 2) !} \\
& \times\left(m^{(3 r-3) / 2}+\frac{3(r-1)(3 r+16)}{100} m^{(3 r-5) / 2}\right. \\
& \left.\quad+\frac{(r-1)}{49 \times 20,000}\left(3969 r^{3}+42615 r^{2}+\ldots\right) m^{(3 r-7) / 2} \ldots\right),
\end{aligned}
$$

whereas

$$
\begin{aligned}
& \frac{1}{6^{r-1} 2^{(r-1) / 2}((r-1) / 2) !}\left(m+\frac{3 r+16}{50}\right)^{(3 r-3) / 2} \\
& =\frac{1}{6^{r-1} 2^{(r-1) / 2}((r-1) / 2) !} \\
& \quad \times\left(m^{(3 r-3) / 2}+\frac{3(r-1)(3 r+16)}{100} m^{(3 r-5) / 2}\right. \\
& \left.\quad+\frac{(r-1)}{49 \times 20,000}\left(3969 r^{3}+35721 r^{2}+\ldots\right) m^{(3 r-7) / 2} \ldots\right) .
\end{aligned}
$$

Thus we need to show that the polynomial

$$
\frac{1}{6^{r-1} 2^{(r-1) / 2}((r-1) / 2) !}\left(m+\frac{3 r+16}{50}\right)^{(3 r-3) / 2}
$$


is a good approximation for $F_{r}(m)$ for $m \geqslant k r$ (say), where $k \geqslant 1$. We can proceed as follows.

Consider the equation

$$
\begin{aligned}
F_{r}(m) & -F_{r}(m-1) \\
& =-\left(\frac{F_{r-2}(m) m^{2}}{3 ! \cdot 2^{2}}+\frac{F_{r-4}(m) m^{4}}{5 ! \cdot 2^{4}}+\cdots+\frac{F_{1}(m) m^{r-1}}{r ! \cdot 2^{r-1}}\right) \text { for } m \geqslant r .
\end{aligned}
$$

We will consider the function

$$
G_{r}(m)=\frac{(-1)^{(r-1) / 2}}{6^{r-1} 2^{(r-1) / 2}((r-1) / 2) !}\left(m+\frac{3 r+16}{50}\right)^{(3 r-3) / 2}
$$

and substitute in both sides of the above equation. We have

$$
\begin{aligned}
& G_{r}(m)-G_{r}(m-1) \\
&= \frac{3(-1)^{(r-1) / 2}}{6^{r-1} 2^{(r-1) / 2}((r-3) / 2) !} \\
& \times\left(\left(m+\frac{3 r+16}{50}\right)^{(3 r-5) / 2}-\frac{1}{2 !}\left(\frac{3 r-5}{2}\right)\left(m+\frac{3 r+16}{50}\right)^{(3 r-7) / 2}\right. \\
&\left.+\frac{1}{3 !}\left(\frac{3 r-5}{2}\right)\left(\frac{3 r-7}{2}\right)\left(m+\frac{3 r+16}{50}-\theta\right)^{(3 r-9) / 2}\right), \\
& \text { where } 0<\theta<1 .
\end{aligned}
$$

The right-hand side becomes, for $s \geqslant 2$,

$$
\begin{aligned}
\frac{3(-1)^{(r-1) / 2}}{6^{r-1} 2^{(r-1) / 2}((r-3) / 2) !} & \\
\times\left(\frac{6}{3 !}\left(m+\frac{3 r+10}{50}\right)^{(3 r-9) / 2}\right. & \cdot m^{2}-\frac{6^{2}}{5 !}\left(\frac{3 r-9}{2}\right)\left(m+\frac{3 r+4}{50}\right)^{(3 r-15) / 2} \cdot m^{4} \\
+\cdots+ & \left.\frac{(-1)^{s+1} 6^{s}\left(\frac{3 r-9}{2}\right) \cdots\left(\frac{3 r-(6 s-3)}{2}\right)}{(2 s+1) !}\right) \\
& \left.\times\left(m+\frac{3 r-(6 s-16)}{50}\right)^{(3 r-(6 s+3)) / 2} \cdot m^{2 s} \ldots\right),
\end{aligned}
$$

where for all $s(3 r-(6 s+3)) / 2$ is positive. Thus all the factors are positive, and the series alternates in sign. We require

$$
\begin{gathered}
\mid s \text { th }|>|(s+1) \text { th } \mid \text { term for } s \geqslant 2 \quad \text { (say), } \\
\therefore(2 s+3)(2 s+2)\left(m+\frac{3 r-(6 s-16)}{50}\right)^{(3 r-(6 s+3)) / 2} \cdot m^{2 s} \\
>6\left(\frac{3 r-(6 s+3)}{2}\right)\left(m+\frac{3 r-(6 s-10)}{50}\right)^{(3 r-(6 s+9)) / 2} \cdot m^{2 s+2} .
\end{gathered}
$$

Adequate if

$$
(2 s+3)(2 s+2)\left(m+\frac{3 r-(6 s-10)}{50}\right)^{3}>3(3 r-(6 s+3)) m^{2}
$$


On the 1.h.s. give $s$ its smallest value when $s=2$ if

$$
42\left(m+\frac{3 r-(6 s-10)}{50}\right)^{3}>3(3 r-(6 s+3)) m^{2} .
$$

If $m \geqslant r$ then r.h.s $<9 m^{3}$ whereas 1.h.s. $>42 m^{3}$. Clearly the first term is larger than the second for $m \geqslant r$. Thus the terms of the series alternate in sign and decrease in absolute magnitude for $m \geqslant r$. Thus the sum of the series is greater than the first two terms but less than the first three. We have that the r.h.s. series

$$
>\left(m+\frac{3 r+10}{50}\right)^{(3 r-9) / 2} \cdot m^{2}-\frac{6^{2}}{5 !}\left(\frac{3 r-9}{2}\right)\left(m+\frac{3 r+4}{50}\right)^{(3 r-15) / 2} \cdot m^{4} \text {. }
$$

We can write this as

$$
\begin{aligned}
(m+ & \left.\frac{3 r+16}{50}-\frac{6}{50}\right)^{(3 r-9) / 2}\left(m+\frac{3 r+16}{50}-\frac{3 r+16}{50}\right)^{2} \\
& -\frac{6^{2}}{5 !}\left(\frac{3 r-9}{2}\right)\left(m+\frac{3 r+16}{50}-\frac{12}{50}\right)^{(3 r-15) / 2} \cdot\left(m+\frac{3 r+16}{50}-\frac{3 r+16}{50}\right)^{4},
\end{aligned}
$$

which is

$$
>\left(m+\frac{3 r+16}{50}\right)^{(3 r-5) / 2}-\left(\frac{3 r-5}{4}\right)\left(m+\frac{3 r+16}{50}\right)^{(3 r-7) / 2} \text { for } m \geqslant r .
$$

We can expand the first three terms in the same way to show that the sum of the series

$$
\begin{aligned}
<\left(m+\frac{3 r+16}{50}\right)^{(3 r-5) / 2} & -\left(\frac{3 r-5}{4}\right)\left(m+\frac{3 r+16}{50}\right)^{(3 r-7) / 2} \\
& +\frac{1}{24}\left(9.79 r^{2}-44.03 r+50.00\right)\left(m+\frac{3 r+16}{50}\right)^{(3 r-9) / 2} .
\end{aligned}
$$

Thus we have that the r.h.s.

$$
\begin{aligned}
& =\frac{3(-1)^{(r-1) / 2}}{6^{r-1} 2^{(r-1) / 2}((r-3) / 2) !} \\
& \quad \times\left(\left(m+\frac{3 r+16}{50}\right)^{(3 r-5) / 2}-\left(\frac{3 r-5}{4}\right)\left(m+\frac{3 r+16}{50}\right)^{(3 r-7) / 2}\right. \\
& \left.\quad+O\left(\frac{1}{24}\left(9.79 r^{2}-44.03 r+50.00\right)\left(m+\frac{3 r+16}{50}\right)^{(3 r-9) / 2}\right)\right),
\end{aligned}
$$

whereas the 1.h.s.

$$
\begin{aligned}
& =\frac{3(-1)^{(r-1) / 2}}{6^{r-1} 2^{(r-1) / 2}((r-3) / 2) !} \\
& \quad \times\left(\left(m+\frac{3 r+16}{50}\right)^{(3 r-5) / 2}-\left(\frac{3 r-5}{4}\right)\left(m+\frac{3 r+16}{50}\right)^{(3 r-7) / 2}\right. \\
& \left.\quad+O\left(\frac{1}{24}\left(9 r^{2}-36 r+35\right)\left(m+\frac{3 r+16}{50}\right)^{(3 r-9) / 2}\right)\right) .
\end{aligned}
$$


Now $F_{r}(m)$ is the function such that

$$
\frac{\text { r.h.s }}{\text { 1.h.s }}=1
$$

We have for $G_{r}(m)$ that

$$
\frac{\text { r.h.s }}{1 . h . s}=\frac{1-\frac{3 r-5}{4(m+(3 r+16) / 50)}+O\left(\frac{9.79 r^{2}-44.03 r+50}{24(m+(3 r+16) / 50)^{2}}\right)}{1-\frac{3 r-5}{4(m+(3 r+16) / 50)}+O\left(\frac{9 r^{2}-36 r+35}{24(m+(3 r+16) / 50)^{2}}\right)}
$$

where $0<O(A)<A$. Let us write $m=k r$ (say), where $k \geqslant 1$ and $r \geqslant 3$. It is easy to show that for $r \geqslant 3$

$$
\begin{aligned}
& 0<O\left(\frac{9.79 r^{2}-44.03 r+50}{24(k r+(3 r+16) / 50)^{2}}\right)<\frac{9.79}{24(k+.06)^{2}}, \\
& 0<O\left(\frac{9 r^{2}-36 r+35}{24(k r+(3 r+16) / 50)^{2}}\right)<\frac{9}{24(k+.06)^{2}} .
\end{aligned}
$$

Hence

$$
\begin{gathered}
\frac{1-\frac{3 r-5}{4(k r+(3 r+16) / 50)}}{1-\frac{3 r-5}{4(k r+(3 r+16) / 50)}+\frac{9}{24(k+.06)^{2}}} \\
<\frac{\text { r.h.s. }}{1 . h . s .}<\frac{1-\frac{3 r-5}{4(k r+(3 r+16) / 50)}+\frac{9.79}{24(k+.06)^{2}}}{1-\frac{3 r-5}{4(k r+(3 r+16) / 50)}} \\
\therefore \frac{1}{1+\frac{9}{24(k+.06)^{2}}\left(\frac{(4 k+.24) r+1.28}{(4 k-2.76) r+6.28}\right)} \\
<\frac{\text { r.h.s. }}{1 . h . s .}<1+\frac{9.79}{24(k+.06)^{2}}\left(\frac{(4 k+.24) r+1.28}{(4 k-2.76) r+6.28}\right) .
\end{gathered}
$$

Now

$$
\begin{aligned}
\max _{r \geqslant 3} & \frac{(4 k+.24) r+1.28}{(4 k-2.76) r+6.28}=\frac{4 k+.24}{4 k-2.76} \\
& \therefore \frac{1}{1+\frac{9}{24(k+.06)^{2}}\left(\frac{4 k+.24}{4 k-2.76}\right)}<\frac{\text { r.h.s }}{1 . h . s}<1+\frac{9.79}{24(k+.06)^{2}}\left(\frac{4 k+.24}{4 k-2.76}\right) .
\end{aligned}
$$

Put $k=2$ (say),

$$
\therefore \text { For } r \leqslant \frac{m}{2} \quad .8779<\frac{\text { r.h.s }}{1 . h . s}<1.1512 \text {. }
$$


Put $k=4$ (say).

$$
\therefore \text { For } r \leqslant \frac{m}{4} \quad .9728<\frac{\text { r.h.s }}{1 . h . s}<1.0304 \text {. }
$$

Clearly as $k \rightarrow \infty$, r.h.s/l.h.s tends to unity, which of course is apparent from the expansion for $F_{r}(m)$ on p. 215. It is thus clear that the function $G_{r}(m)$ is a good approximation for $F_{r}(m)$ in the sense defined above. That is, the ratio is close to unity. Thus we can say that for $r \leqslant m / 2, G_{r}(m)$ is a 'good' approximation for $F_{r}(m)$. Thus, for $r \leqslant m / 2$ at least, the functions $F_{r}(m)$ must be of constant sign as the functions $G_{r}(m)$ certainly are, and the series will be an alternating one. The leading polynomials listed to $r=21$ are of constant sign for $r \leqslant m$ that is with $k=1$ a property which is most probably general but is not proved here. In any event, it is only the leading terms that we require, and $r$ of the order $m / 2$ is quite sufficient. Thus for $r \leqslant m / 2$ we can approximate $F_{r}(m)$ with $G_{r}(m)$ such that if $a_{r}$ is the $r$ th term of the series expansion for $q_{m}(r)$, where $r=1,2,3, \ldots$, then

$$
\begin{aligned}
\frac{a_{r+1}}{a_{r}} & =\frac{F_{2 r+1}}{F_{2 r-1}} \cdot \frac{\{m-(2 r-1)\}(m-2 r)}{(n+m(m-3) / 4)^{2}} \\
& \doteqdot \frac{1}{72 r}\left(m+\frac{6 r+19}{50}\right)^{3} \frac{(m-2 r)}{(n+m(m-3) / 4)^{2}}<1 \\
& \quad \text { if }\left(n+\frac{m(m-3)}{4}\right)>\frac{(m-2 r)}{\sqrt{72 r}}\left(m+\frac{6 r+19}{50}\right)^{3 / 2} .
\end{aligned}
$$

Now the right-hand side for fixed $m$ and positive $r$ is monotonically decreasing. Thus the terms constantly decrease in absolute value if, putting $r=1$, we have

$$
\left(n+\frac{m(m-3)}{4}\right)>\frac{(m-2)}{\sqrt{72}}\left(m+\frac{1}{2}\right)^{3 / 2} \text {. }
$$

Clearly $n>m^{5 / 2} / \sqrt{72}$ is adequate. For comparison the two estimates are

$$
\begin{array}{lllr}
m=20 & n>112 & n>210 \\
m=30 & n>354 & n>581 \\
m=50 & n>1443 & n>2084 \\
m=100 & n>9212 & n>11,786
\end{array}
$$

The series can still be of value even when $n$ is smaller than the above bounds as the terms must ultimately begin to decrease, as the following example shows. Consider $P_{50}(1000)$.

\section{We have}

$\begin{array}{lr}.369652 \times 10^{30} & .369652 \times 10^{30} \\ .617024 \times 10^{30} & -.247372 \times 10^{30} \\ .480117 \times 10^{30} & .232745 \times 10^{30} \\ .231361 \times 10^{30} & .138390 \times 10^{28} \\ .773674 \times 10^{29} & .787513 \times 10^{29} \\ .190671 \times 10^{29} & .596842 \times 10^{29} \\ .359008 \times 10^{28} & .632743 \times 10^{29} \\ .528360 \times 10^{27} & .627460 \times 10^{29} \\ .616781 \times 10^{26} & .628076 \times 10^{29}\end{array}$


The underestimate will initially be negative and thus trivial, but if enough terms are retained an improved estimate is possible. We have in this case that

$$
.627460 \times 10^{29}<p_{50}(1000)<.628076 \times 10^{29},
$$

whereas $P_{50}(1000)=.628023 \times 10^{29}$. If the next two terms are included, we have

$$
\begin{array}{ll}
.576293 \times 10^{25} & .628019 \times 10^{29} \\
.433098 \times 10^{24} & .628023 \times 10^{29}
\end{array}
$$

On the other hand $n$ cannot in general be too small, as the following argument will indicate. The terms of the series begin to decrease in absolute value when

$$
\sqrt{72}\left(n+\frac{m(m-3)}{4}\right)>\frac{(m-2 r)}{\sqrt{r}}\left(m+\frac{6 r+19}{50}\right)^{3 / 2} \text {. }
$$

The r.h.s is monotonically decreasing for $r=1,2,3, \ldots$. Thus we need to determine the smallest $r$ such that

$$
\sqrt{72}\left(n+\frac{m(m-3)}{4}\right)=\frac{(m-2 r) m^{3 / 2}}{\sqrt{r}}\left(1+\frac{6 r+19}{50 m}\right)^{3 / 2} .
$$

Now the maximum value of $1+(6 r+19) / 50 m=1+(6 m+19) / 50 m$. Thus

$$
\underset{r}{\operatorname{Max}}\left(1+\frac{6 r+19}{50 m}\right)^{3 / 2}<(1 \cdot 5)^{3 / 2} \text {. }
$$

Thus if we consider the simpler relationship

$$
\frac{(m-2 r)}{\sqrt{r}}=\frac{\sqrt{72}}{m^{3 / 2}}\left(n+\frac{m(m-3)}{4}\right)=X \quad \text { (say) }
$$

we have that

$$
\begin{aligned}
& r=\frac{\left(X^{2}+4 m\right) \pm \sqrt{\left(X^{2}+4 m\right)^{2}-16 m^{2}}}{8}, \\
\therefore \quad & r \doteqdot \frac{m^{2}}{X^{2}+4 m} \text { as } \frac{16 m^{2}}{\left(X^{2}+4 m\right)^{2}}<1, \\
\therefore \quad & r \doteqdot \frac{m^{5}}{72\left(n+\frac{m(m-3)}{4}\right)^{2}+4 m^{4}}, \text { where } r=1,2,3,4, \ldots
\end{aligned}
$$

Now $p(m)=p_{m}(2 m)$, and thus we have in this case that

$$
r \doteqdot \frac{m^{5}}{72\left(2 m+\frac{m(m-3)}{4}\right)^{2}+4 m^{4}} \doteqdot \frac{2 m}{17} .
$$

Thus $r \rightarrow \infty$ with $m$, and the series is thus of no value for calculating $p(m)$.

One final example using the above approximation for $r$.

6. Consider $P_{50}(500)$. We have that $r \doteqdot 2.8$. Thus the third term in the series is approximately the largest in absolute magnitude. In fact, the largest is the fourth, as the following results show. 


$\begin{array}{lr}.329499 \times 10^{22} & .329499 \times 10^{22} \\ .117201 \times 10^{23} & -.842513 \times 10^{22} \\ .194333 \times 10^{23} & .110081 \times 10^{23} \\ .199553 \times 10^{23} & -.894714 \times 10^{22} \\ .142198 \times 10^{23} & .527271 \times 10^{22} \\ .746776 \times 10^{22} & -.219505 \times 10^{22} \\ .299625 \times 10^{22} & .801205 \times 10^{21} \\ .939667 \times 10^{21} & -.138462 \times 10^{21} \\ .233746 \times 10^{21} & .952837 \times 10^{20} \\ .465398 \times 10^{20} & .487439 \times 10^{20} \\ .745310 \times 10^{19} & .561970 \times 10^{20}\end{array}$

Thus $.487 \times 10^{20}<p_{50}(500)<.562 \times 10^{20}$, whereas $P_{50}(500)=.553301 \times 10^{20}$.

7. Conclusion. The alternating series expansion for $q_{m}(n)$ provides an effective means for determining both upper and lower bounds when $n>m^{5 / 2} / \sqrt{72}$.

Department of Mathematics

North East London Polytechnic

Barking Precinct

Longbridge Road

Dagenham, Essex RM8 2AS, England

1. G. E. Andrews, The Theory of Partitions, Addison-Wesley, Reading, Mass., 1976.

2. L. E. Dickson, History of the Theory of Numbers, Vol. 2, Chelsea, New York, 1966.

3. H. GuPTA, Tables of Partitions, The Royal Society Mathematical Tables, Vol. 4, Cambridge, 1958.

4. G. J. RIEGER,“Über partitionen,” Math. Ann., v. 138, 1959, pp. 356-362. 